\title{
LATCHING MICRO ELECTROMAGNETIC RELAYS
}

\author{
M. Ruan and J. Shen \\ Dept. of Electrical Engineering and Center for Solid State Electronics Research, Arizona State University, Tempe, \\ AZ 85286. " Correspondence: Email: jshen@ asu.edu; Phone: (480)-965-9517 \\ C. B. Wheeler \\ Microlab, Inc., 6401 E. Hummingbird Ln., Paradise Valley, AZ 85253-3608
}

\begin{abstract}
This paper describes a new type of latching micro magnetic relay that has recently been demonstrated. The device is based on preferential magnetization of a permalloy cantilever in a permanent external magnetic field. Switching between two stable states is accomplished by a short current pulse through an integrated coil underneath the cantilever. Some key features are summarized as follows. (1) Latching (bistable); (2) low energy consumption during switching ( $\$ 93 \mu \mathrm{J}$, switching current $\sim 60 \mathrm{~mA}$, minimum switching pulse width $\sim 0.2 \mathrm{~ms}$ ); (3) low voltage operation ( $\leqslant 5 \mathrm{~V}$ ); (4) maximum DC current $>500 \mathrm{~mA}$; (5) capable of various switch configurations [single-pole-single-throw (SPST), multi-polesingle-throw (MPST), or multi-pole-double-throw (MPDT)]; (6) low contact resistance $(\$ 50 \mathrm{~m} \Omega) ;(7)$ operation in ambient environment; (8) lifetime expected to be comparable to other micro relays, (9) batch fabrication using planar processing methods.
\end{abstract}

\section{INTRODUCTION}

Latching relays are widely used in applications such as aerospace, RF communications, and portable electronics, where power consumption must be minimized. Conventional electromechanical relays typically operate by energizing an elecromagnet that actuates a soft magnetic armature to make or break a contact. When the magnet is deenergized, a spring restores the armature to its quiecent position. Recently, similar principles have been applied to microelectromechanical (MEMS) relays using the microlectronics fabrication methods [1-4]. Application of MEMS technology to relays should enable drastic reductions in fabrication costs, physical size and design complexity. To our knowledge, a latching magnetic MEMS relay has not been demonstrated.

In this work, the preferential magnetization of a permalloy cantilever is utilized to achieve two stable states. In a constant, nearly perpendicular magnetic field, a cantilever can have either a clockwise or a counter-clockwise torque depending on the angle between the cantilever and the field, which leads to the bistability. To switch the relay, a second magnetic field (generated by a current pulse through a coil in this case) realigns the magnetization of the cantilever and changes the direction of the magnetic torque, causing the cantilever to flip.

\section{PRINCTPLE OF OPERATION}

The basic structure of the microswitch is illustrated in Fig. 1. The device consists of a cantilever, an embedded planar coil, a permanent magnet, and the necessary electrical contacts. The cantilever is a two-layer composite consisting of a soft magnetic material (e.g., $\mathrm{NiFe}$ permalloy) on its top side and a highly conductive material, such as $\mathrm{Au}$, forms the bottom surface. The cantilever is fixed at one end and is free to deflect at the opposite end (Figs. 1 and $2 a$ ). A second design variation that has also been explored has a cantilever supported by torsion flexures from the two sides (Fig. 2b). The contact end to the right of the cantilever can be deflected up or down by applying a current through the coil. When it is in the "down" position, the cantilever makes electrical contact with the bottom conductor, and the switch is "on" ("closed"); when the contact end is "up", the switch is "off" ("opened"). The permanent magnet holds the cantilever in either the "up" or the "down" position after switching, making the device a latching relay.
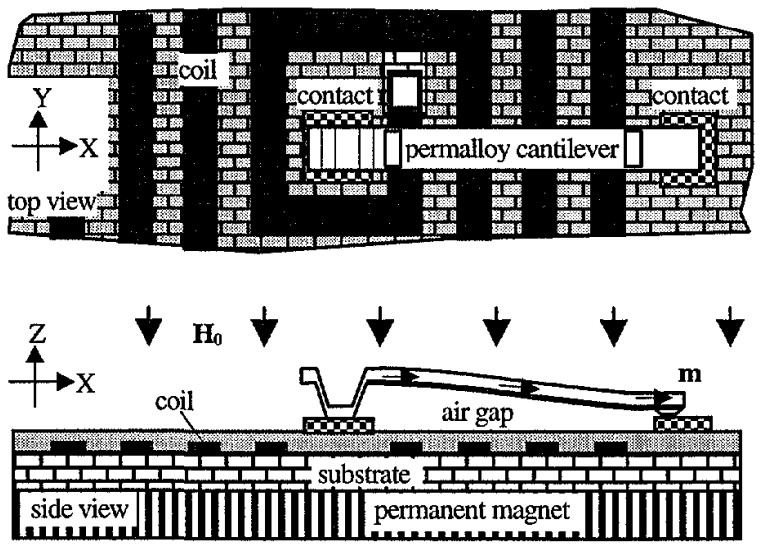

Figure 1. Top and side views of the latching relay. Planar coils are integrated on the same substrate underneath the cantilever. A permanent magnet provides the constant magnetic field $\mathrm{H}_{0}$ which holds the cantilever to either of its stable states.
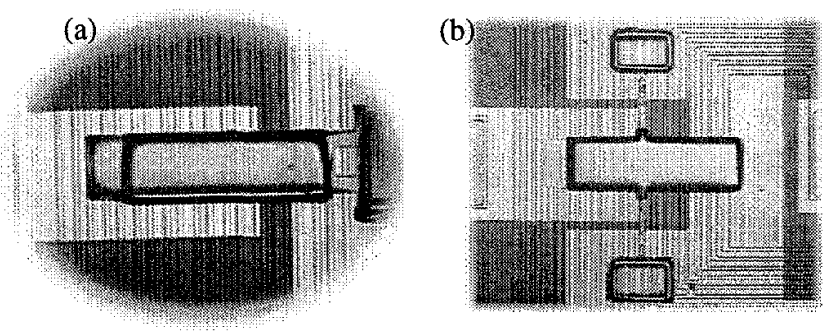

Figure 2. Photographs of the fabricated latching relays. Two types of design [(a) one-end fixed; (b) center-hinged] have been investigated and both types are successfully demonstrated. Typical beam size: $800 \mu \mathrm{m} \times 200 \mu \mathrm{m} \times 25 \mu \mathrm{m}$.

\section{(i) Method to produce bistability}

When the length $L$ of a permalloy cantilever is much larger than its thickness $t$ and width $w$, the direction along its long axis $(L)$ becomes the preferred direction for magnetization (easy axis). When such a cantilever is placed in a uniform permanent magnetic field, a torque is exerted on the beam. The torque can be either clockwise or counterclockwise, depending on the initial orientation 
of the beam with respect to the magnetic field. When the angle $(\alpha)$ between the beam axis $(\xi)$ and the external field $\left(\boldsymbol{H}_{0}\right)$ is smaller than $90^{\circ}$, the torque is counterclockwise; and when $\alpha$ is larger than $90^{\circ}$, the torque is clockwise (Fig. 3). The bi-directional torque arises because of the bi-directional magnetization (by $\boldsymbol{H}_{0}$ ) of the beam (from left to right when $\alpha<90^{\circ}$, and from right to left when $\alpha>90^{\circ}$ ). Due to the torque, the beam tends to align with the external magnetic field $\left(\boldsymbol{H}_{0}\right)$. Ilowever, when a mechanical force (such as the elastic torque of the beam, a physical stopper, etc.) preempts to the total realignment with $\boldsymbol{H}_{0}$, two stable positions ("up" and "down") are available, which forms the basis of latching in the relay.

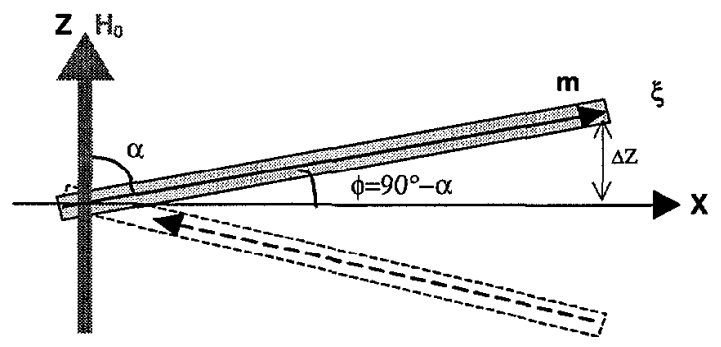

Figure 3. Schernatic drawings of the relay for discussions. $\boldsymbol{H}_{0}$ is the magnetic field provided by the permanent magnet, $m$ is the magnetic moment of the cantilever, $\alpha$ is the angle between $H_{0}$ and $\boldsymbol{m}, \phi=90^{\circ}-\alpha$, and $\Delta z$ is the deflection height at the end.

\section{(ii) Electrical switching}

If the bi-directional magnetization along the easy axis of the beam arising from $\boldsymbol{H}_{0}$ can be momentarily reversed by applying a second magnetic field to overcome the influence of $\left(\boldsymbol{H}_{0}\right)$, then it is possible to achieve a switchable latching relay. This scenario is realized by situating a planar coil under the cantilever to produce the required temporary switching field. The planar coil geometry was chosen because it is relatively simple to fabricate, though other structures (such as a wrap-around type) are also possible. The magnetic field $\left(\boldsymbol{H}_{\text {coil }}\right)$ lines generated by a short current pulse loop around the coil. It is mainly the $\xi$-component (along the beam, see Fig. 3) of this field that is used to rcoricnt the magnctization in the beam. The direction of the coil current determines whether a positive or a negative $\xi$-field component is generated. After switching, the permanent magnetic field holds the beam in this state until the next switching event is encountered. Since the $\xi$ component of the coil-generated field $\left(H_{c o i l-\xi}\right)$ only needs to be momentarily larger than the $\xi$-component $\left[H_{0 \xi} \sim H_{0} \cos (\alpha)=\right.$ $\left.H_{0} \sin (\phi), \alpha=90^{\circ}-\phi\right]$ of the permanent magnetic field and $\phi$ is typically very small $\left(\phi \leqslant 5^{\circ}\right)$, switching current and power can be very low, which is an important consideration in micro relay design.

\section{DESIGN AND FABRICATION}

\section{(1) Torque and bistability}

Assuming a uniform magnetization of the beam, the magnetic moment can be expressed as $\boldsymbol{m}=\boldsymbol{M V}$, where $\boldsymbol{M}$ is the magnetization in the beam and is determined by the external magnetic field $\left(\boldsymbol{H}_{\boldsymbol{\theta}}\right)$, the beam geometry and magnetic properties, and $V$ is the volume of the beam. The magnetic bending moment $\tau_{\mathrm{m}}$ is obtained using

$$
\tau_{m}=\boldsymbol{m} \times \boldsymbol{B}_{0}=\mu_{0} \boldsymbol{m} \times \boldsymbol{H}_{0},
$$

For the cantilever design with one fixed end, a key question is whether the magnetic force is large enough to overcome the mechanical force so that the beam will deflect and make contact with the contact pad on the substrate. ${ }^{1}$ According to the elasticity theory, the elastic torque needed to cause a deflection $\Delta z$ at the free end of the cantilever is [5]

$$
\tau_{e}=w t^{3} \Delta z E / 4 L^{2}
$$

where $E$ is the Young's modulus of the beam. For a beam of $L=600 \mu \mathrm{m}, w=10 \mu \mathrm{m}$ and $t=1 \mu \mathrm{m}$, and assuming $E=2 \times 10^{11} \mathrm{~N}-\mathrm{m}^{-2}$ for the permalloy [6], we have for a deflection of $\Delta z=12 \mu \mathrm{m}$ $\left(\phi=1.6^{\circ}\right)$,

$$
\tau_{e}=1.8 \times 10^{-11} \mathrm{~N}-\mathrm{m}
$$

According to (1), we numerically calculated the magnetic torque in a magnetic field of 0.037 Tesla and obtained a value of

$$
\tau_{m}=8.4 \times 10^{-10} \mathrm{~N}-\mathrm{m}
$$

ncarly 4.7 times as large as $\tau_{e}$. In the calculation, we have used the permalloy B-H curves and included the demagnetization factors. In principle, the weight of the beam causes the beam to bend downward, but this bending can be shown to be negligibly small.

\section{(2) Switching field}

The current-induced coil magnetic field has both an $\mathrm{X}$ and a $\mathrm{Z}$ component. It is mainly the $\mathrm{X}$ component that is used for switching.

The magnetic field distribution around the planar coil can be calculated using the Biot-Savart Law:

$$
\vec{B}_{c o i l}=\int d \vec{B}=\frac{\mu_{0}}{4 \pi} \int \frac{I \cdot d \vec{l} \times \vec{r}}{r^{3}}
$$

A numerical routine was developed to calculate the field distribution. The calculated magnetic field at positions $\mathrm{Z}=4 \mu \mathrm{m}$ above the $X$-axis is calculated and shown in Fig. 4. $B_{z}$ and $B_{x}$ are the field components along $\mathrm{Z}$ - and $\mathrm{X}$-axis respectively. In the calculation, we used 25 turns for the coil. The spacing between the adjacent turns is $\Delta x=20 \mu \mathrm{m}$. The current in the coil is $50 \mathrm{~mA}$. Because the beam lies in the positive side of the $X$-axis, we show in more detail the $\mathrm{X}$-component of the magnetic field in that region in Fig. 4 for several different heights $(\mathrm{z}=4-80 \mu \mathrm{m})$. The oscillatory behavior in $B$ for small $\mathrm{Z}(=4 \mu \mathrm{m})$ is due to the proximity effect and the discreteness of the coil line separation. The average field strength is in the approximate range between 0.001 Tesla and 0.002 Tesla.

To evaluate under what conditions the coil current can switch the relay, the magnetization process needs to be examined in more detail. As mentioned above, when the aspect ratio of a beam is large (i.e., $L>w>>t$ ), the magnetization primarily aligns in the direction of $L$. Much smaller components of the magnetization also exist along the directions of $w$ and $t$, but can be neglected due to the large demagnetization in these directions. Thus the beam's magnetization is primarily determined by the external field component along its long axis. To switch the relay, the coil

\footnotetext{
${ }^{1}$ This is not an issue for the hinged-type design because the mechanical torsion can be very small in that case.
} 
current-induced magnetic field $\left(B_{\text {coil- } \xi}\right)$ must overcome the external field $\left(B_{0 \xi}\right)$ along the cantilever's length, at least momentarily.
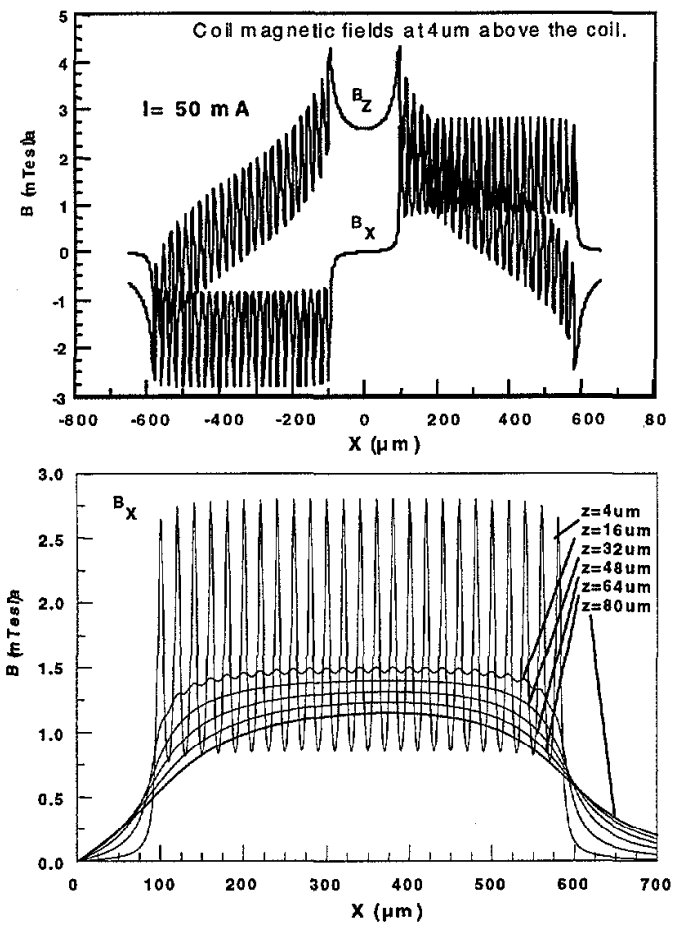

Figure 4. Calculated magnetic flux generated by a current of $50 \mathrm{~mA}$ though the planar coil. The $x$-component of the magnetic field is used to switch the magnetization orientation, and thus the relay. The oscillatory behaviors in $\boldsymbol{B}$ are due to the proximity gaps between the coil lines. The results are used to determine the necessary magnitude of switching current and the number of turns in the coil in order to switch the relay.

Consider the center-hinged type cantilever as an example to estimate the magnetic field strengths. We assume $L=800 \mu \mathrm{m}$, $w=200 \mu \mathrm{m}, t=25 \mu \mathrm{m}$, the stage height (air gap spacing) $h=12 \mu \mathrm{m}$, and the external magnetic field $B_{0}=0.037$ Tesla (equivalent to $H_{0}=$ 370 Oersted). The key purpose is to verify that $B_{\text {coil }-\xi}>B_{05}$ when the cantilever is in the "close" position (the result also applies to the "open" case because of the symmetry). $B_{0 \xi}$ depends on both $B_{0}$ and the angle $\alpha\left[B_{0 \xi}=B_{0} \cos (\alpha)\right]$. When the cantilever is in the "close" position, we obtain $\alpha=180^{\circ}-\arccos (12 / 0.5 L)=180^{\circ}$ $\arccos (12 / 400)=91.72^{\circ}$. So, we have $B_{0}=-0.0011$ Tesla. On the other hand, $B_{\text {coil- } \xi} \approx B_{\text {coil- } \mathrm{X}} \sin (\alpha) \approx B_{\text {coil- } \mathrm{X}}$, and its average value is around 0.0014 Tesla at the height of $12 \mu \mathrm{m}$ (Fig. 4). We can see that under these conditions, a $50 \mathrm{~mA}$ current in the planar coil is able to generate a magnetic field large enough to reverse the magnetization in the cantilever and switch the relay. The analysis applies to other cases where the supporting torsion flexure is not located exactly at the center of the cantilever, and the switching current can be different between the two switching events. Another point worth noting is that because the current-induced coil magnetic field only needs to overcome the $\xi$ component of the external field $\left(B_{0}\right)$ and not the $\boldsymbol{Z}$ component, the switching current can be relatively small.

\section{(3) Fabrication}

Conventional surface micromachining techniques were used to fabricate the latching relays. The device was fabricated on a Si- substrate covered with an insulating dielectric. A Ag coil was formed by e-beam evaporation and wet etching. A polyimide layer was spin-cast on to cover the coils. Then bottom Au contact layers were deposited and patterned. Photoresist was used as a sacrificial layer. The cantilever was formed by electroplating NiFe permalloy on a Au seed layer. The cantilever was then released, and the relay diced and finally mounted on a permanent magnet.

\section{RESULTS AND DISCUSSIONS}

Latching micro relays of both the center-hinge and fixed-end type have successfully been demonstrated. A number of different beam dimensions - ranging from $10 \mu \mathrm{m} \leq w \leq 600 \mu \mathrm{m}, 80 \mu \mathrm{m} \leq L$ $\leq 1000 \mu \mathrm{m}, 10 \mu \mathrm{m} \leq t \leq 30 \mu \mathrm{m}$, and $12 \mu \mathrm{m} \leq h \leq 18 \mu \mathrm{m}$ - have been evaluated for both cantilever types. A 20-turn coil was used for all the devices.

Photographs of two types of micro magnetic relays (hinged and fixed-end) are shown in Fig. 2. Both types are fully functional; demonstrating both latching operation and electrical switching.

\section{(1) Resistance}

Resistance was measured using the four-probe method. For a typical hinged-type relay ( $L=1000 \mu \mathrm{m}, w=500 \mu \mathrm{m}, t=30 \mu \mathrm{m}, h=18$ $\mu \mathrm{m})$, the measured lead-to-lead resistance was $52.8 \mathrm{~m} \Omega$ under a 410 Oersted permanent magnet field with a switching current of $118 \mathrm{~mA}$

\section{(2) Switching characteristics}

Electrical switching characteristics are shown in Fig. 5. A waveform generator (HP33120A) was used to produce the coil current pulses for switching. A DC voltage of $3.2 \mathrm{~V}$ was applied to the relay with a load resistor $\left(R_{L}=5 \mathrm{k} \Omega\right)$ in series. Both the input signal $\left(V_{i n}\right)$ and the relay signal $\left(V_{R}\right.$ on the load) were recorded on
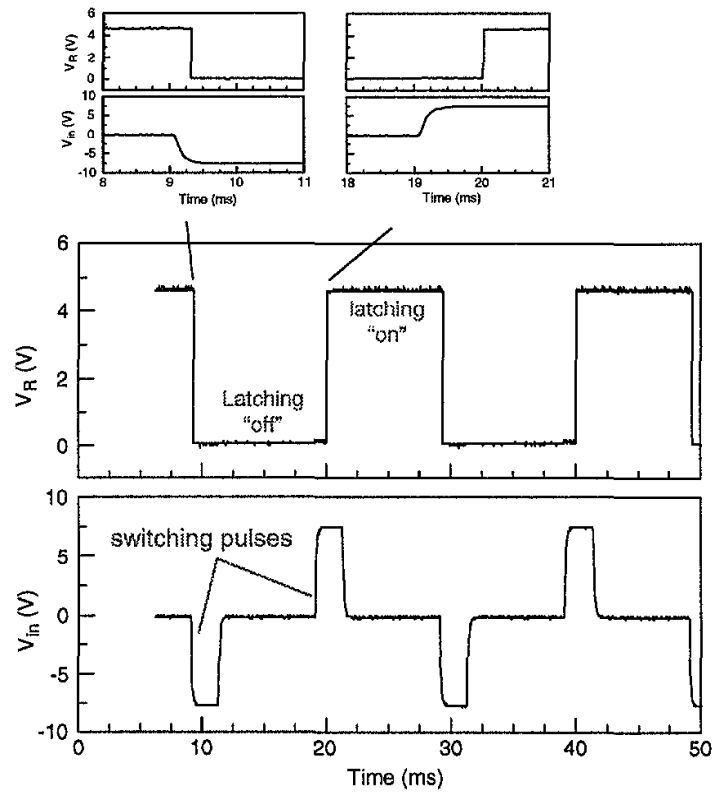

Figure 5. Typical switching characteristics of the latching relay. A square-wave-type current pulse (peak amplitude of $100 \mathrm{~mA}$ ) was used to switch the relay. As can be seen, the relay latches after switching. The top enlarged plots show the on and off transient characteristics. Beam dimensions: $800 \mu \mathrm{m} \times 200 \mu \mathrm{m} \times 25 \mu \mathrm{m}$. 
an HP Oscilloscope (HP54615B). The input pulse width was $3 \mathrm{~ms}$ (the shortest measured input pulse width is $0.2 \mathrm{~ms}$ ) and the duty cycle was $20 \mathrm{~ms}$. The magnitude of the driving pulse was $7.5 \mathrm{~V}$, which corresponds to a current of $100 \mathrm{~mA}$ with a coil resistance of $75 \Omega$. As can be seen, a negative current pulse through the coil lurns off the relay and a positive pulse tums on the relay. Between the pulses, the relay latches in a stable state without any power consumption. The turn-on and -off transient characteristics are shown in the enlarged plots in Fig. 5. Oscillations in the circuit are also observed (Fig. 6) and can be due to the parasitic capacitive and inductive elements in the device and/or measurement setup.

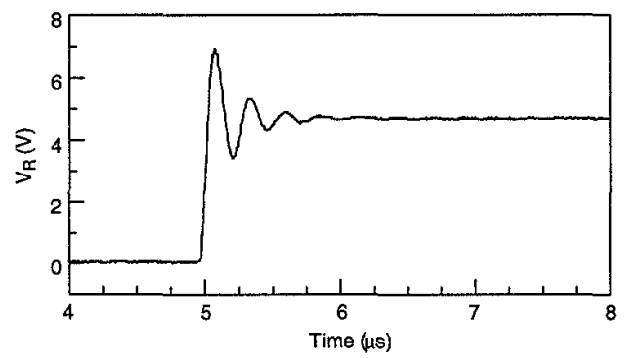

Figure 6. Oscillation behaviors are also observed at the turn-on. Parasitic LC elements in the measurement setup and/or the device may contribute to the oscillation.

\section{(3) Reliability}

The maximum DC current is $>500 \mathrm{~mA}$. Lifetime tests were performed on a center-hinged-type relay with the following dimensions: $L=1000 \mu \mathrm{m}, w=500 \mu \mathrm{m}, t=30 \mu \mathrm{m}$, air gap $h=12 \mu \mathrm{m}$. The permanent magnetic field strength was 370 Oersted and the switching current was $100 \mathrm{~mA}$. No mechanical or electrical degradation (permanent contact resistance change) were observed after the relay was switched for $4.8 \times 10^{6}$ cycles in ambient conditions with an "on" current of $240 \mu \mathrm{A}$. Momentary resistance changes were occasionally observed during test and might have been due to particles in the air. Further evaluations are under way to better understand the long-term contact properties.

\section{SUMMARY}

This paper describes a new type of latching micro magnetic relay that has recently been demonstrated. The device is based on preferential magnetization of a permalloy cantilever in a permanent external magnetic field. Switching between two stable states is accomplished by a short current pulse through an integrated coil underneath the cantilever. Some key features are summarized as follows. (1) I atching (bistable); (2) low energy consumption during switching ( $\$ 93 \mu \mathrm{J}$, switching current $\sim 60 \mathrm{~mA}$, minimum switching pulse width $\sim 0.2 \mathrm{~ms}$ ); (3) low voltage operation $\leqslant 5 \mathrm{~V}$ ); (4) maximum DC current $>500 \mathrm{~mA}$; (5) capable of various switch configurations [single-pole-single-throw (SPST), multi-polesingle-throw (MPST), or multi-pole-double-throw (MPDT)]; (6) low contact resistance ( $\leqslant 50 \mathrm{~m} \Omega$ ); $(7)$ operation in ambient environment; (8) lifetime expected to be comparable to other micro relays, (9) batch fabrication using planar processing methods.

The most significant advantage of this relay is its bistability and consequent elimination of power consumption in the quiescent states. Other desirable properties (such as low contact resistance, low operation voltage, high off-state isolation, simplicity of design and fabrication, fast switching and large actuation force) also make the device a good candidate for applications in automatic testing equipment, smart interconnects, and other fields that require latching relays.

Acknoledgement: JS and CBW acknowledge partial support from Air Force Research Laboratory/VS, 3550 Aberdeen Ave. S. E., Kirtland AFB, NM 87117-5776. The authors also acknowledge valuable technical contributions from Dr. Warren Wilson - AFRL.

\section{REFERENCES}

[1] J. W. Judy and R. S. Muller, "Magnetically actuated, addressable microstructures," J. Microelectromechanical Systems 6, 249 (1997)

[2] W. P. Taylor, O. Brand, and M. G. Allen, "Fully integrated magnetically actuated micromachined relays," IEEE J. Microelectromech. Syst. 7, 181 (1998).

[3] J. A. Wright, Y.-C. Tai, and G. Lilienthal, "A magnetostatic MEMS switch for DC brushless motor commutation," Proceedings: Solid-State Sensor and Actuator Workshop 1998, Hilton Head Island, South Carolina, pp. 304-307 (1998).

[4] H. A. C. Tilmans, E. Fullin, H. Ziad, M. D. J. Van de Peer, J. Kesters, E. Van Geffen, J. Bergqvist, M. Pantus, K. Baert and F. Naso, "A fully-packaged electromagnetic microrelay", Proceed. $12^{\text {th }}$ Ann. Int. Conf. MEMS, pp 25-30 (1999).

[5] P. P. Benham, R. J. Crawford, and C. G. Armstrong, Mechanics of Engineering Materials, 2nd edition (Longman, Essex, 1996), pp. 189.

[6] The Young's modulus for the permalloy was not available to the authors at the time of writing this paper. The value for nickel was therefore used. See CRC Handbook of Chemistry and Physics, 78th edition, pp. 12-204. 